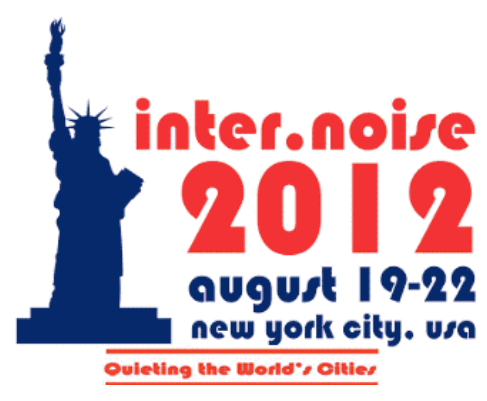

\title{
Noise policy - integration with climate and natural resource policies
}

\author{
Tor Kihlman ${ }^{\text {a) }}$ \\ Chalmers University of Technology \\ 41296 Gothenburg \\ Sweden
}

Climate change policy is difficult. Noise policy is even more difficult because of its complexity. There is such a wide range of society's processes/activities in which noise problems are integrated. There are win/win situations. Lower noise emissions facilitate healthy quiet compact cities. Compact cities save land use and fuel, have typical shorter travelling distances, lower speeds are possible. Barrier effects can be reduced. Walking and bicycling can be promoted. There are conflicts. Demands upon reduced fuel consumption and cleaner exhaust gases from road vehicles and jet engines make the noise emission problems tougher. Traffic safety demands on roads may collide with ways to reduce the noise emissions. Politicians in national and international bodies call noise a local problem which, however, local politicians cannot solve if not the source levels get reduced which is an area for international agreements. National industrial interests delay progress towards quieter products. Economic sub-optimization lead to unfortunate end results. To get a really quieter world demands that planners, builders, industries, etc. all do more. Speed policy is an important area. Few politicians understand the real complexity of the noise problems. We, noise professionals, have to spend more time on teaching and influencing them.

\section{INTRODUCTION}

This paper does not give any examples upon how noise policy has been integrated in other policy areas. It discusses where such integration is desirable and should be of mutual advantage. For the time being, such integration may be utopic but appears necessary for effective solutions or even real progress. The paper is limited to problems linked to transportation.

In general, different problem areas are treated isolated from each other. This is the easiest way in decision-making. However, to solve complex environmental problems a holistic approach may be preferable or even necessary and will in the end be cheaper.

a) email: tor.kihlman@chalmers.se 
Two environmental problems are discussed here; climate change which is a difficult problem and noise which is a still more difficult and complex problem. Interrelations between these should be identified, taken into account and made best use of in the development of solutions.

In the case climate, it is clear for the politicians and the public that a long-term strategy is necessary. The acute problem is the lack of an effective policy. Nor is the problem continuously followed up by strong actions. In the case noise, the need for a strategy is less understood or admitted by the politicians.

It is very demanding to solve anyone of these two complex problems but until they are lifted to a strategic level and taken seriously, continuously, long-term it is definitely impossible to make real progress. In the case of noise, both emission and immission policies are important.

There are relations between these two environmental problems. Some conditions create win/win situations, other conditions give rise to conflicts. It is essential to realize this especially on a strategic level.

\section{GENERAL APPROACH TO SOLVE ENVIRONMENTAL PROBLEMS}

Many environmental problems have been treated and solved successfully in isolation from other problems. This is especially the case where it has been possible to find solutions without sacrificing essential utilities for the public. Some examples:

Products of all kinds of industrial and consumer products are today recycled. It reduces the "garbage mountain" and the need for new raw material. It is a modest sacrifice for the consumer and is accepted by the public.

Filters for polluted water and gases have been introduced. We have got much cleaner rivers and lakes and cleaner air in the cities. Exhaust gases from ICEs (Internal Combustion Engines) do no longer cause the same smog problems in cities as they did a few decades ago. We can drive our cars without severe restrictions for short-term air pollution reasons.

Several poisonous or dangerous substances have been forbidden. CFCs have been phased out and replaced by other substances with less effects upon the ozone layer. DDT, PCB and many other products have become forbidden. These actions have been possible and accepted by the public as other substances giving the same utility or service have existed or been developed.

Even though this type of approach has been successfully used for many problems it appears insufficient for the more complex problems. Utilities and comfort may get in danger.

\section{THE CLIMATE PROBLEM}

Climate change policy is more difficult than the above-mentioned environmental problems. Suppose that climate change is real and man-induced and that the emission of large amounts of the climate gases carbon dioxide and methane is the main cause. This is the assumption in this paper. To limit these gases as much as IPCC (International Panel for Climate Change) claims 
necessary is very demanding. Radical cuts in emission of climate gases are claimed to be necessary within a very short time to limit the heating of the planet to +2 degrees centigrade. Even worse, it is now questioned by IPCC whether it is not already too late to stop global warming at +2 degrees. If so, it is still more urgent to act strategic and speed up the political process.

To meet the climate change threat demands a long-term strategy and continuous political leadership. Short-term problems must not take over or delay progress and actions. Nevertheless, it has already been allowed to do so in the shadow of the economic crisis.

The public has not yet perceived the severe effects of climate change. Few perceive the climate change other than through the media reports concerning the development of total climate gas emissions and a hitherto small increase in global temperature. Climate change may be the reason for some extreme weather conditions, which have occurred during the last years.

It appears evident that reductions of the total emissions of carbon dioxide and methane are needed in order to slow down and stop the climate change. However, this implies very uncomfortable decisions. Utilities for the public are endangered with consequences for our daily life.

\section{THE NOISE PROBLEM}

Noise policy is in many respects even more difficult than climate policy because of its complexity. However, in contrast to the climate change problem, the noise is here already and has been so since long time with obvious severe effects upon health and well-being. Many citizens are affected directly and personally by the noise. Yet, few realize the severe effects upon public health. The noise causes cardio-vascular diseases resulting in hypertension and heart attacks. WHO, the World Health Organization, has recently published two reports on these effects, [1], [2].

The traffic noise in Europe is estimated to cause more than one million lost healthy life years per year in the population and the number of fatal heart attacks caused by the traffic noise is of the same order as the fatal traffic accidents. WHO has given recommendations on limits both for day and night both for short-term and long-term. These targets are set with strong scientific backing. They are very demanding to achieve. Typical levels today lie far above these recommended levels. It will be difficult for EU Member States to follow these recommendations but it should also be difficult for these Member States to ignore the severe problem. The WHO reports give imperative arguments for an ambitious noise policy.

Few politicians understand the real complexity of the noise problems. It has also been typical for many politicians to refer to noise as a local problem. In some senses this may be correct, but the problems cannot be solved at a local level. On the contrary, it demands long-term strategies on national and international levels because the environmental noise problems cannot be solved unless the emissions get much reduced. This means a substantial reduction of maximum allowed noise emission for products that are sold worldwide, such as airplanes, cars, trucks, two-wheelers, tyres. Lower emission limits demand international agreements. Problems may then arise due to national industrial interests. Many parties need to take coordinated actions in order to get substantial improvements. The lead times from decision to noticeable effects in 
terms of a quieter environment span over several decades which of course implies long-term strategies and good follow-up. If the steps towards more stringent limits are small and taken with too long times between consecutive steps, we may never get to the quieter environment; the small steps may be compensated by the continuous increase in traffic.

\section{COULD INTEGRATED POLICIES GIVE ADVANTAGES?}

Both the climate problem and the noise problem are complex and demand long-term policies. There are links between the climate issue and the community noise issue. The noise problems are integrated in a wide range of processes and activities in the society.

There are win/win situations. Lower noise emissions facilitate healthy, quiet compact cities. Compact cities save land and fuel, have typical shorter travelling distances, lower speeds are possible. The conditions for public transportation are good as well as for walking and bicycling.

There are conflicts. Demands upon reduced fuel consumption and cleaner exhaust gases for ICEs and jet engines make the noise emission problems tougher. The demand for higher efficiency of ICEs for road vehicles leads to demand for higher compression ratios which in turn leads to more difficult noise problems. For airplanes, there are trade-offs between noise reduction, fuel consumption and NOX emissions. Noise reduction measures often lead to increased weight.

For road vehicles, not only the engine noise is the problem, also the rolling noise is problematic. There are many requirements on tyres and road surfaces to be fulfilled. Low rolling resistance and good wet grip is needed. There may be conflicts here with low noise emissions. If top speeds are reduced, it should be easier to meet the requirements. Lower speeds are beneficial for traffic safety. Lower average speeds lead to lower fuel consumption and lower noise emissions.

\section{THE ROAD TRAFFIC NOISE PROBLEM IN SOME DETAIL}

The majority of the persons exposed to high road traffic noise levels live in urban areas. Equivalent levels of $65 \mathrm{~dB}$ and more are there common along major roads and busy streets. Sprawling does not help; in some respects it rather worsens the situation. In the sprawled city, the longer distances demand higher typical speeds. In order to reduce the noise, it is needed both to reduce the emissions and to regard town and building planning to reduce the immissions.

The noise levels outside a high fraction of the homes are much too high to correspond to a healthy environment. Even with the best city and building planning, there is a gap of $10 \mathrm{~dB}$ or more between the noise emission from the ordinary traffic and what is possible to achieve by best planning to get a reasonably healthy environment. We have a serious gap between emission requirements and healthy immissions. The way to close this gap is to reduce the noise emission per vehicle in ordinary traffic by $10 \mathrm{~dB}$; a time-demanding and tough task. The electric cars, when they totally dominate the car fleet, may be part of the solution for personal transportation at speeds below $30 \mathrm{~km} / \mathrm{h}$. 
Different cities, compact as well as sprawled, are surprisingly equal in terms of traffic noise power expressed as emission per unit urban area. This is because the traffic work (the total traffic in the city) expressed as vehicle*km per unit time and urban area is surprisingly equal in most major cities. These characteristics of city traffic have been discussed in a couple of papers, [3], [4]. The data imply that the average traffic noise emission per unit urban area has a spread of approx. only $3 \mathrm{~dB}$ and is independent of population density. There seems to be an urban cultural law of traffic leading to this result. Cf also Zahavi's law [5].

From a general point of view, sprawled cities are no quieter than compact ones but the noise problems are different and involve different challenges. The sprawled city may have enclaves with excellent quiet environment but the necessary high-speed main arteries which link these enclaves give rise to very noisy environments. Who suffers? We may have a democratic problem! The compact city can have blocks which offer a high degree of quietness but the distance from these to the busy streets are short and the noise exposure of the buildings facing the streets get high also if the traffic speed is low.

With regard to climate and noise policies and in considering the links, the compact city can be a win/win situation, but to make the city healthy and pleasant the noise levels need to be reduced in relation to what is common today.

\section{CONCLUSIONS}

Noise policy is challenging. It will take a long time to achieve a substantially quieter world with less health effects caused by noise. Adequate policies are urgently needed. They must involve participation by many parties, the industry, the town planners, the builders and many others.

Noise is an integrated part or effect of almost every major activity in the society. This also makes it extremely complex. It also means that there are links between the noise policy and other complex policy areas. Such links should be observed and taken into account in the needed longterm policies. One important link to be observed is between noise and climate. Here we have both conflicts and win/win situations.

Global warming is supposed to be caused by the emission of climate gases. Much of these come from traffic. Compact cities lead to less use of fuel for transportation and they save land. They should be promoted in an ambitious climate policy. Such compact cities if well planned can create conditions for quiet and thereby healthy environments provided that the noise emissions from the traffic are kept low. This is quite possible, as the compact city demands only moderate speeds in the traffic system. Quiet transportation modes, such as well-designed individual and public transportation systems, bicycles and walking can serve the citizens. This demands international agreements on emission limits overcoming national industrial interests.

But on the other hand, if the noise emission requirements of the transportation system are neglected, the compact city may result in a very noisy and unhealthy environment. Our policymakers often do not understand the complexity of the noise issue. We, noise professionals, have to spend more time on teaching and influencing them. 


\section{ACKNOWLEDGEMENT}

I gratefully acknowledge the Adlerbert Research Foundation in Gothenburg for economic support.

\section{REFERENCES}

1. "Night Noise Guidelines for Europe”, WHO Regional Office for Europe, (2009)

2. "Burden of Disease from environmental noise, Quantification of healthy life years lost in Europe", WHO Regional Office for Europe, (2011)

3. T. Kihlman, and W. Kropp, "Limits to the Noise Limits", Intl Congress on Acoustics, Seattle, (1998)

4. T. Kihlman, and W. Kropp, "Soundscapes in cities, limits to the noise limits" Forum Acusticum 99, Berlin, (1999)

5. Y. Zahavi, and M. Ryan. "Stability of Travel Components Over Time" Transportation Research Record, 750: 19-26, (1980) 